DUK-TH-94-68

IASSNS-HEP-94/23

April, 1994

\title{
String Theory on K3 Surfaces
}

\author{
Paul S. Aspinwall, \\ School of Natural Sciences, \\ Institute for Advanced Study, \\ Princeton, NJ 08540 \\ David R. Morrison \\ Department of Mathematics, \\ Box 90320, \\ Duke University, \\ Durham, NC 27708-0320
}

\begin{abstract}
The moduli space of $N=(4,4)$ string theories with a K3 target space is determined, establishing in particular that the discrete symmetry group is the full integral orthogonal group of an even unimodular lattice of signature $(4,20)$. The method combines an analysis of the classical theory of K3 moduli spaces with mirror symmetry. A description of the moduli space is also presented from the viewpoint of quantum geometry, and consequences are drawn concerning mirror symmetry for algebraic K3 surfaces.
\end{abstract}




\section{Introduction}

In recent years, Calabi-Yau manifolds have received great attention in the string literature. This is mainly because compactification on such spaces may be used to reduce the number of dimensions in models built from the intrinsically ten dimensional critical superstring [1]. The focus has largely been on the case of complex dimension $d=3$, since the corresponding compactification is to four space-time dimensions. (It is also the case that, in many respects, within topological field theory on Calabi-Yau target spaces the case $d=3$ plays a special rôle [2, 3].) However, one might expect interesting properties for other values of $d$ as well.

In this paper we analyze the case $d=2$ whose special features derive from the fact that the topology of the target must be either a torus or a K3 surface. The classification of toroidal

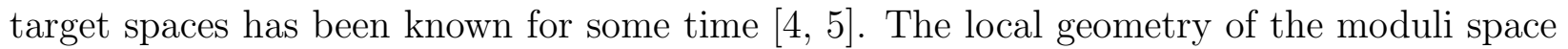
of conformal field theories with K3 target space topology was understood shortly afterwards [6] and although some aspects of the global form of the moduli space have been conjectured and studied [6, 0, 8], more precise statements concerning this question have remained elusive.

We will use mirror symmetry to address this question of the form of the moduli space. We find that, given a couple of minor assumptions, combining a $\sigma$-model analysis near the field theory limit with the study of a mirror symmetry transformation is sufficient to give the precise form of the moduli space. This paper is not intended to provide the full mathematical exposition of the analysis required - for that the reader is referred to [9]. Here we only give a brief summary of the methods used and conclusions reached by the analysis.

The analysis of the moduli space of string theories on a K3 surface differs markedly from that of the more familiar Calabi-Yau moduli spaces related to string compactifications to four dimensions. Firstly, in studying the case where the target space $X$ is a Calabi-Yau threefold, the assumption $h^{2,0}=0$ is usually made. Under this assumption, the deformations of complex structure of $X$ and of the (complexified) Kähler form "decouple" to give the complete moduli space a local product structure [10] (at least over generic points in the moduli space). These two types of deformations may then be studied independently. For a K3 surface we have $h^{2,0}=1$ and the above structure is lost. We are thus forced to analyze deformations of complex structure and deformations of Kähler form together.

In another respect however the K3 case is simpler - the $N=(2,2)$ superconformal invariance of a string with Calabi-Yau target space is extended to $N=(4,4)$ supersymmetry in the case $d=2$ [11]. This is equivalent to the geometric statement that a Calabi-Yau manifold in complex dimension two admits a hyperkähler metric. This $N=(4,4)$ structure serves to fix the local form of the moduli space completely. This can be contrasted with the case $d=3$ where the extended chiral algebra [12] has yet to provide much insight into the classification of string theories.

As in the case $d=3$, the local dimension of the moduli space of conformal field theories 
can be matched to the dimension of the moduli space of the geometrical objects representing the target space if we regard the "geometrical object" as including, in addition to the target space metric, the specification of a $B$-field, i.e., an element of the second real (de Rham) cohomology group of the target. In the case $d=3$, the $B$-field naturally combined with the Kähler form to provide a complexified Kähler form. In the case of K3 this is clearly impossible - $B$ lives in a 22-dimensional space whereas the dimension of the space of Kähler forms varies with the complex structure but is at most 20. Despite this fact we will still find that a beautiful structure arises when the $B$-field is included in the moduli space.

At the topological level, mirror symmetry might appear rather trivial for a K3 surface since the mirror of a K3 surface (equipped with metric and $B$-field) is another such K3 surface. However, the geometric data of the mirror K3 surface thus obtained is not, in general, isomorphic to the original. Thus the mirror map acts as a non-trivial automorphism on the "classical" moduli space of K3 surfaces (i.e., the $\sigma$-model moduli space of metrics and $B$-fields). The mirror map in this context was first studied long ago in 13 and some of the observations in that paper lead to some of the methods used here.

Although the purpose of this paper is to concentrate on the case where the target space is a K3 surface, it should be noted that much of what follows applies to any target space with $h^{2,0}=1$. The only steps in the following argument which will not be directly applicable to the determination of the moduli space of this more general case are the explicit form of the moduli space of Einstein metrics, and the particular self-mirror K3 theory used.

In section 2 we will present the outline of the construction of the moduli space of $N=(4,4)$ and $N=(2,2)$ theories. In section 3 we discuss the interpretation of the moduli space in terms of the space of total cohomology of K3. In section A, some aspects of mirror symmetry on algebraic K3 surfaces are discussed.

\section{The Moduli Space}

Let us first fix some notations. $\Gamma \backslash G / H$ denotes the double coset space resulting from dividing the group $G$ by the right-action of $H$ and left-action of $\Gamma$. ( $G$ and $H$ will always be continuous Lie groups and $\Gamma$ will be discrete.) $\Lambda^{a, b}$ denotes the unique (up to isomorphism) even selfdual lattice in $(a+b)$-dimensional space with signature $(a, b)$, when $a b \neq 0$. Basis vectors may be taken such that the inner product has the following form:

$$
\Lambda^{a, b}:\langle,\rangle \cong \underbrace{\left(-E_{8}\right) \oplus\left(-E_{8}\right) \oplus \ldots}_{n \text { times }} \oplus H \oplus H \oplus \ldots,
$$


where $b-a=8 n$ (one uses $E_{8}$ in place of $-E_{8}$ when $b-a<0$ ), $E_{8}$ is the Cartan matrix of the Lie algebra $E_{8}$, and $H$ is the hyperbolic plane:

$$
H=\left(\begin{array}{ll}
0 & 1 \\
1 & 0
\end{array}\right) .
$$

We will use $X$ to denote a specific smooth K3 surface. One can show that the intersection pairing on $H^{2}(X, \mathbb{Z})$ gives it the structure of a lattice isomorphic to $\Lambda^{3,19}$ (see for example $[14]$. Let $\mathbb{R}^{a, b}$ be a $(a+b)$-dimensional space with an inner product of signature $(a, b)$. $O(a, b)$ is the orthogonal group on $\mathbb{R}^{a, b}$ and $O\left(\Lambda^{a, b}\right)$ is the subgroup preserving $\Lambda^{a, b} \subset \mathbb{R}^{a, b}$. Let

$$
\mathscr{T}^{a, b}=O(a, b) /(O(a) \times O(b)) .
$$

We can identify $\mathscr{T}^{a, b}$ as the set of space-like $a$-planes in $\mathbb{R}^{a, b}$ (i.e., $a$-planes on which the inner product is positive-definite). $\mathscr{T}^{a, b}$ can also be regarded as one of the two connected components of the set of oriented space-like $a$-planes. Under this latter interpretation, we can write

$$
\mathscr{T}^{a, b}=O^{+}(a, b) /(S O(a) \times O(b)),
$$

where $O^{+}(a, b)$ is the index 2 subgroup of $O(a, b)$ with the same connected components as $S O(a) \times O(b)$.

It was shown in [6] using arguments from supergravity [15] that the required moduli space, $\mathscr{M}$, of conformal field theories on a K3 surface is locally of the form $\mathscr{T}^{4,20}$. This is probably best understood from the argument presented in [16], which we now review. The $N=(4,4)$ superconformal algebra contains affine $S U(2)$ algebras in both the left and right sectors. This symmetry acts on the marginal operators spanning the tangent spaces of $\mathscr{M}$. The existence of such a symmetry restricts the form of the holonomy group of the Zamolodchikov metric of $\mathscr{M}$. The restriction is so severe, in fact, that it then follows from Berger's classification [17] of holonomy groups that either $\mathscr{M}$ is locally isomorphic to a quaternionic symmetric space, or that the holonomy is reducible. Moreover, the non-flatness of the Zamolodchikov metric is enough to rule out the reducible holonomy case. A simple analysis of any conformal field theory giving rise to a K3 target space tells us that $\mathscr{M}$ has real dimension 80 [6, 11]. This, together with the known classification of quaternionic symmetric spaces (cf. [18]), completes the proof.

\footnotetext{
${ }^{1}$ One way to do this count is as follows: the space of complex structures has complex dimension 20 (real dimension 40), the space of Kähler forms has real dimension 20, and the space of $B$-fields has real dimension 22. However, for each Ricci-flat metric on the K3 surface there is an $S^{2}$ of complex structures, so the overall dimension count is $40+20+22-2=80$.
} 
Let us now make two assumptions about the form of $\mathscr{M}$, both of which we consider to be quite reasonable. Firstly we assume $\mathscr{M}$ is geodesically complete $]^{2}$ and secondly we assume $\mathscr{M}$ to be Hausdorff. While some non-Hausdorff moduli spaces have appeared in string theory [19], this happened in the context of a target space of indefinite signature. There is no reason to believe that something as unpleasant as this should happen in the case of a K3 surface. It then follows that

$$
\mathscr{M} \cong \Gamma \backslash \mathscr{T}^{4,20}
$$

for some group $\Gamma$ acting discretely on $\mathscr{T}^{4,20}$. Thus to complete the description of $\mathscr{M}$, we only need to find $\Gamma$.

We begin our determination of $\Gamma$ by analyzing the classical form of the moduli space of K3 surfaces. Using the techniques of [20], one may decompose $\mathscr{T}^{4,20}$ as

$$
\mathscr{T}^{4,20} \cong \mathbb{R}_{+} \times \mathscr{T}^{3,19} \times \mathbb{R}^{3,19}
$$

where $\mathbb{R}_{+}$is the half-line of positive real numbers which we parameterize by $\lambda$. The decomposition depends on a choice of null vector $v \in \mathbb{R}^{4,20}$, and the space $v^{\perp} / \mathbb{R} v$ provides the $\mathbb{R}^{3,19}$ on which $O(3,19)$ acts and on which $\mathscr{T}^{3,19}$ is based. The space $\mathscr{T}^{3,19}$ is known to be isomorphic to the Teichmüller space for Einstein metrics of volume one on a K3 surface (and their orbifold limits) [21, 22, 23]. It is natural then to identify $\lambda$ as giving the size of the K3 surface and $\mathbb{R}^{3,19}$ as the moduli space of $B$-fields on the target space. These identifications may be established by looking at the metrics on the above mentioned spaces. The Zamolodchikov metric on $\mathscr{T}^{4,20}$ is known to be the left-invariant metric. From [24] this induces a metric on each of the terms on the right hand of (6) where this should now be viewed as a "warped product", i.e., the metric does not respect the product structure. One may now show (using [23]) that the metrics induced are those given precisely by the extended WeylPetersson metric in the sense of [25]. Such an isometric identification allows us to identify every point in $\mathscr{T}^{4,20}$ with an Einstein metric on a K3 surface (or orbifold metric) together with a $B$-field. This can be taken as another version of the statement that the non-linear $\sigma$-model is exactly conformally invariant on a K3 surface with Ricci-flat metric [26, 27, 28]. It also shows that the Zamolodchikov metric and Weyl-Petersson metric coincide exactly on the moduli space - a fact which in general $N=(2,2)$ theories holds only to leading order in the large radius limit 29 .

The moduli space of smooth Einstein metrics of volume one on a K3 surface $X$ is determined in 23] to be $\Gamma_{0} \backslash \mathscr{T}^{3,19}-\mathscr{Z}$, where $\Gamma_{0}=\operatorname{Diff}(X) / \operatorname{Diff}^{0}(X)$ is the group of components of the diffeomorphism group of $X$, and $\mathscr{Z}$ is the space of orbifold metrics. (It is generally believed that such orbifolds should be included when considering a string target space [30];

\footnotetext{
${ }^{2}$ As we will point out below, we must include orbifold points in our moduli space in order to ensure this geodesic completeness.
} 
if we include them, we get the geodesically complete space $\Gamma_{0} \backslash \mathscr{T}^{3,19}$.) The discrete group $\Gamma_{0}$ is determined in [31, 32, 33] to coincide with the group

$$
O^{+}\left(\Lambda^{3,19}\right):=O\left(\Lambda^{3,19}\right) \cap O^{+}(3,19),
$$

which has index 2 in $O\left(\Lambda^{3,19}\right)$. The "missing" $\mathbb{Z}_{2}$ in $O\left(\Lambda^{3,19}\right)$ may be generated by $-I$ acting on $\mathbb{R}^{3,19}$.

We also know that the non-linear $\sigma$-model on a Calabi-Yau manifold is invariant under all translations $B \rightarrow B+v$, where $v \in H^{2}(X, \mathbb{Z})$. Thus we should divide the space $\mathbb{R}^{3,19}$ of $B$-fields by additive translations by $\Lambda^{3,19}$. (As an abstract group, these additive translations simply form a $\mathbb{Z}^{22}$.) Furthermore one may consider complex conjugation of the target space. If one considers such a complex conjugation for a $\sigma$-model one sees that the transformation $B \rightarrow-B$ is required in addition to the conjugation of the complex structure of the target. Such a transformation may be represented by $-I$ and thus generates the missing $\mathbb{Z}_{2}$ from above. Therefore, in terms of the decomposition (6) one sees a group $O\left(\Lambda^{3,19}\right) \ltimes \Lambda^{3,19}$ (which is the full space-group of $\Lambda^{3,19}$ ) of identifications that should be made on the right-hand side and thus on $\mathscr{T}^{4,20}$. Thus we obtain

$$
\Gamma \supseteq O\left(\Lambda^{3,19}\right) \ltimes \Lambda^{3,19}
$$

This is the maximal set of identifications that can be made from classical geometry. Any further statement requires some quantum geometry. The mirror construction of [34] provides us with such a tool. First we need the clarify the meaning of the mirror map in the context of $N=(4,4)$ theories. Mirror symmetry reverses the sign of a $U(1)$ charge derived from an $N=2$ chiral algebra. Now an $N=4$ chiral algebra contains an $S U(2)$ affine subalgebra, which is larger than the $U(1)$ affine subalgebra of an $N=2$ theory. Given an $N=4$ theory, though, we may choose an $N=2$ subalgebra of the $N=4$ algebra by specifying the corresponding $U(1) \subset S U(2)$. Each $N=4$ theory thus gives rise to an $S^{2} \cong S U(2) / U(1)$ of $N=2$ theories. This leads to construction of the moduli space of $N=(2,2)$ theories of string on a K3, which we denote by $\mathscr{M}_{q \bar{q}}$, as a fibre bundle

$$
\pi: \mathscr{M}_{q \bar{q}} \rightarrow \mathscr{M}
$$

with fibre $S^{2} \times S^{2}$. Each point in $\mathscr{M}_{q \bar{q}}$ corresponds to an $N=(4,4)$ theory on which a particular $N=(2,2)$ structure has been chosen; the subscripts $q$ and $\bar{q}$ denote charges with respect to the left and right $U(1)$ currents respectively within the $N=(2,2)$ theory.

A (left) mirror map on the corresponding Teichmüller space $\mathscr{T}_{q \bar{q}}^{4,20}$ of $N=(2,2)$ theories is a map $\mu: \mathscr{T}_{q \bar{q}}^{4,20} \rightarrow \mathscr{T}_{q \bar{q}}^{4,20}$ with the property that, for all $S \in \mathscr{T}_{q \bar{q}}^{4,20}$, the $N=(2,2)$ theories at $S$ and $\mu(S)$ are isomorphic, but with a switch of charge assignments $(q, \bar{q}) \leftrightarrow(-q, \bar{q})$. 


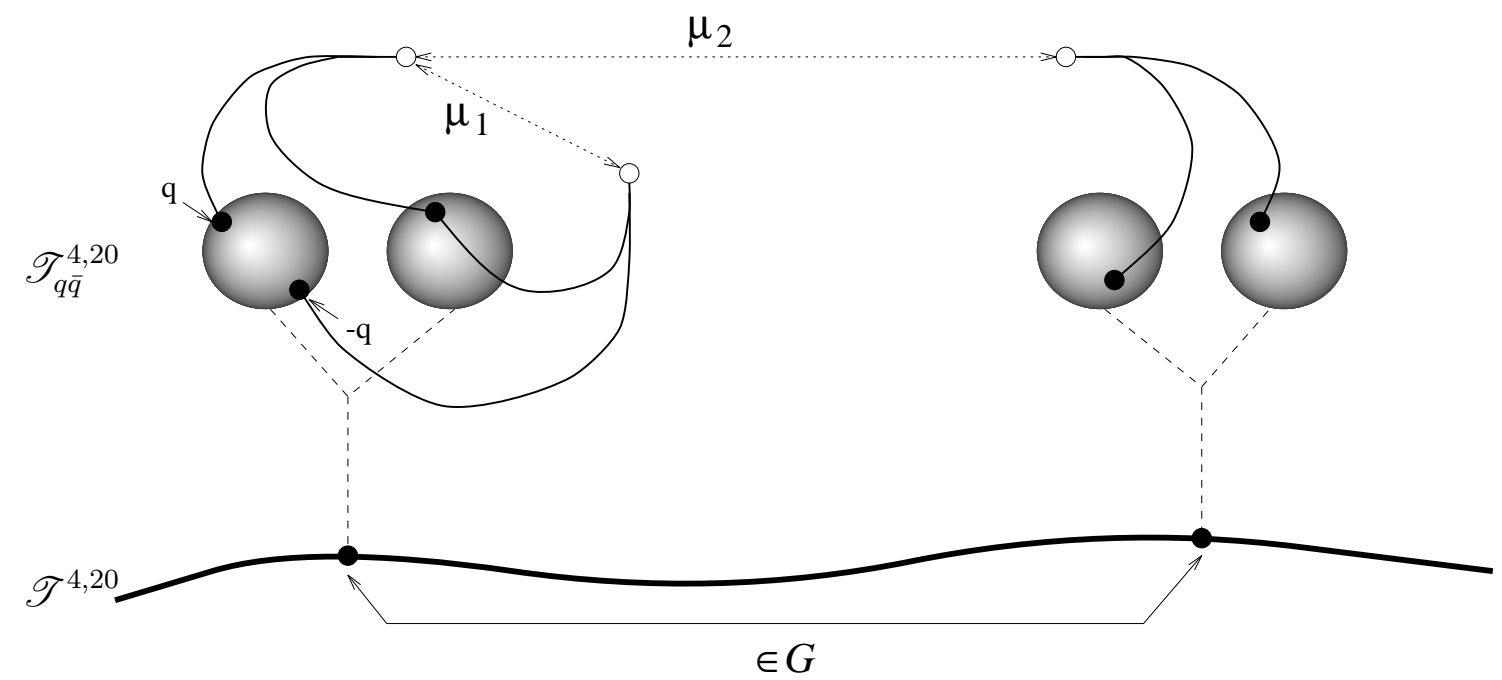

Figure 1: Trivial and nontrivial mirror maps. $\mathscr{T}_{q \bar{q}}^{4,20}$ is an $\left(S^{2} \times S^{2}\right)$-bundle over $\mathscr{T}^{4,20}$ as shown. A theory (shown as an open circle) is associated with a pair of points, one on each sphere. In this figure $\mu_{1}$ is trivial and $\mu_{2}$ is nontrivial.

We also define right mirror maps which switch $(q, \bar{q}) \leftrightarrow(q,-\bar{q})$. Such a map on $\mathscr{T}_{q \bar{q}}^{4,20}$ can be pushed down to a map $\bar{\mu}$ on the Teichmüller space $\mathscr{T}^{4,20}$ — since two theories which are mirrors as $N=(2,2)$ theories are isomorphic as $N=(4,4)$ theories, any mirror map within $\mathscr{T}^{4,20}$ should give rise to an element of $\Gamma$. Note that some mirror maps on $\mathscr{T}_{q \bar{q}}^{4,20}$ may be trivial on $\mathscr{T}^{4,20}$, in the sense that they induce the identity element of $\Gamma$. Such maps relate pairs of points in $\mathscr{T}_{q \bar{q}}^{4,20}$ which lie over the same point in the base space $\mathscr{T}^{4,20}$. What will be of more interest are the nontrivial mirror maps which map to a nontrivial element of $\Gamma$. The difference between trivial and nontrivial maps is shown schematically in figure 11.

We now consider an example of a generically nontrivial mirror map with a fixed point on $\mathscr{T}^{4,20}$. Given the Gepner model [35] associated via [36] to the K3 hypersurface

$$
X_{0}^{2}+X_{1}^{3}+X_{2}^{7}+X_{3}^{42}=0
$$

in the weighted projective space $\mathbb{P}_{\{21,14,6,1\}}^{3}$ we find the mirror as an orbifold of the original space by the method of [34]. The orbifolding group thus found is trivial and so this theory is its own mirror. The fact that this theory is self-mirror is also related to some issues regarding Arnold's "strange duality" observed in [13]. This mirror map acts non-trivially on the marginal operators of this theory and thus on the tangent bundle of $\mathscr{T}^{4,20}$. By analyzing this action, it is possible to show that there is a lattice $\Lambda^{4,20} \subset \mathbb{R}^{4,20}$ such that the induced 
automorphism $\bar{\mu}$ of $\mathscr{T}^{4,20}$ lies in $O\left(\Lambda^{4,20}\right)$. In fact, there is a decomposition

$$
\Lambda^{4,20} \cong \Lambda^{2,10} \oplus \Lambda^{2,10}
$$

such that $\bar{\mu}$ acts by simply exchanging the two terms on the right-hand side. The decomposition can be chosen so that one of the $\Lambda^{2,10}$ lattices in (11) is a sublattice of the $\Lambda^{3,19}$ lattice on which the classical $O\left(\Lambda^{3,19}\right)$ symmetry of (8) acts.

We have thus found the explicit form of another generator of $\Gamma$. A straight-forward but somewhat involved calculation then shows that the classical symmetries, $O\left(\Lambda^{3,19}\right) \ltimes \Lambda^{3,19}$, and the above $\mathbb{Z}_{2}$ mirror map generate the whole of $O\left(\Lambda^{4,20}\right)$. That is, we have

$$
\Gamma \supseteq O\left(\Lambda^{4,20}\right)
$$

But now from [37], which establishes that $O\left(\Lambda^{4,20}\right)$ is a maximal discrete group acting on $\mathscr{T}^{4,20}$ to yield a Hausdorff quotient, it follows that

$$
\Gamma \cong O\left(\Lambda^{4,20}\right)
$$

completing the proof that the moduli space has the precise form

$$
\mathscr{M}=O\left(\Lambda^{4,20}\right) \backslash O(4,20) /(O(4) \times O(20))
$$

(as had been speculated by Seiberg [6]).

To summarize, we have shown that the full group of identifications to be made on the Teichmüller space is generated by the following:

1. Classical identifications made for the moduli space of complex structures.

2. The integral $B$-field shifts.

3. Complex conjugation (together with a change in sign of the $B$-field).

4. The mirror map.

One should note that this is also true for the case $d=1$ where the target space must be a torus [38.

It is interesting to note that this moduli space $\mathscr{M} \cong \Gamma \backslash \mathscr{T}^{4,20}$ is precisely that which one would obtain for toroidal compactifications of the heterotic string down to six-dimensional spacetime using the methods of $\mathbb{4}$. This equivalence between the moduli space of K3 compactifications and toroidal compactifications (for the relevant strings) was established locally in [6] but it is unclear why they turn out to be globally isomorphic. 


\section{The Space of Total Cohomology}

The derivation of the moduli space $\mathscr{M}$ in the previous section and [9] is somewhat unpleasant and yet produces a beautifully symmetric result. This is a consequence of having derived the moduli space using the ideas of classical geometry necessitating the intermediate step of equation (8). Now that we have the moduli space we may reinterpret it from the standpoint of quantum geometry in a more symmetric way.

First let us quickly review the form of the moduli space of complex structures on a classical K3 surface. Consider a non-vanishing holomorphic 2-form $\Omega$ on $X$ representing an element of $H^{2,0}(X)$. Let us specify a "marking" of $X$, i.e., a basis $\gamma_{i}, i=1, \ldots, 22$ of $H_{2}(X, \mathbb{Z})$ determining an isomorphism of that lattice with $\Lambda^{3,19}$. The global Torelli theorem [39, 40, 21] essentially says that the complex structure of $X$ is expressed uniquely in terms of the periods

$$
\int_{\gamma_{i}} \Omega
$$

up to a change of basis of $H_{2}(X, \mathbb{Z})$. To state this more precisely, we write $\Omega=\xi+i \eta$, where $\xi, \eta \in H^{2}(X, \mathbb{R}) \cong \Lambda^{3,19} \otimes_{\mathbb{Z}} \mathbb{R}$. The Hodge-Riemann bilinear relations assert that

$$
\begin{aligned}
& \langle\Omega, \Omega\rangle=\int_{X} \Omega \wedge \Omega=0 \\
& \langle\Omega, \bar{\Omega}\rangle=\int_{X} d V\|\Omega\|^{2}>0 .
\end{aligned}
$$

This implies that the oriented 2-plane $\xi \wedge \eta$ in $H^{2}(X, \mathbb{R})$ spanned by $\xi$ and $\eta$ is spacelike, i.e., has positive definite metric. In fact, specifying $\Omega$ up to multiplication by $\mathbb{C}^{*}$ (which determines the Hodge structure) is equivalent to specifying the oriented 2-plane $\xi \wedge \eta$. Now the moduli space of oriented space-like 2-planes in $H^{2}(X, \mathbb{R}) \cong \mathbb{R}^{3,19}$ is given by $O(3,19) /(S O(2) \times O(1,19))$. When we mod out by diffeomorphisms, we would expect to obtain a description of the moduli space of complex structures on a K3 surface as an open subset of

$$
O^{+}\left(\Lambda^{3,19}\right) \backslash O(3,19) /(S O(2) \times O(1,19))
$$

(and we would expect to get the entire space (17) if we include orbifold complex structures). Actually, there are some technical difficulties in interpreting (17) as a moduli space, but the interpretation is essentially correct (see 21] for more precise statements).

The extension of this to string theory follows immediately. The space $\mathscr{T}^{4,20}$ is one connected component of the set of oriented space-like 4 -planes in $\mathbb{R}^{4,20}$. The space $\mathbb{R}^{4,20}$ can be identified with the space $H^{*}(X, \mathbb{R})$ of all real cohomology groups on $X$ - the inner product is obtained by supplementing the intersection pairing on $H^{2}(X, \mathbb{R})$ by a pairing on 
$H^{0}(X, \mathbb{R}) \oplus H^{4}(X, \mathbb{R})$ which has matrix (2) on the standard generators of those two spaces (i.e., the class of a point, and the class of the entire space $X)$. The natural lattice $H^{*}(X, \mathbb{Z})$ inside of $H^{*}(X, \mathbb{R}) \cong \mathbb{R}^{4,20}$ inherits an integer-valued pairing from this inner product, giving it the structure $\Lambda^{4,20}$. An $N=(4,4)$ theory on a K3 surface is thus specified uniquely by an oriented space-like 4-plane in $H^{*}(X, \mathbb{R})$ (belonging to the correct component) where the 4-plane is located relative to the $H^{*}(X, \mathbb{Z})$ lattice.

This picture becomes clearer when we go to the $N=(2,2)$ moduli space. Now the moduli space, $\mathscr{M}_{q \bar{q}}$, is an $\left(S^{2} \times S^{2}\right)$-bundle over $\mathscr{M}$. In fact one may show that the Teichmüller space takes the form

$$
\mathscr{T}_{q \bar{q}}^{4,20}=O^{+}(4,20) /(S O(2) \times S O(2) \times O(20)) .
$$

In the language of the previous paragraph, this is one component of the space of orthogonal pairs of oriented space-like 2 -planes in $\mathbb{R}^{4,20}$. That is, we not only specify an oriented spacelike 4-plane but we give it an internal structure of being spanned by two orthogonal oriented 2-planes. We may now specify these oriented 2-planes in terms of complex vectors as in the classical case. Namely, define $\Omega$ and $\mho$ as arbitrary elements of $H^{*}(X, \mathbb{C})\left(\bmod \mathbb{C}^{*}\right)$ such that

$$
\begin{aligned}
& \langle\Omega, \Omega\rangle=\langle\Omega, \mho\rangle=\langle\mho, \mho\rangle=\langle\bar{\Omega}, \mho\rangle=0, \\
& \langle\Omega, \bar{\Omega}\rangle>0, \quad\langle\mho, \bar{\mho}\rangle>0 .
\end{aligned}
$$

The space of such vectors gives precisely the Teichmüller space required. Thus we may identify the moduli space of $N=(2,2)$ theories on a K3 surface as being the space of all possible choices of $\Omega$ and $\mho$ subject to (19) where the positions of these vectors are located relative to $H^{*}(X, \mathbb{Z})$. This statement may be considered to be the quantum version of the global Torelli theorem.

As explained earlier, mirror maps will act on the Teichmüller space $\mathscr{T}_{q \bar{q}}^{4,20}$. In fact a nontrivial mirror map may be identified with the interchange $\Omega \leftrightarrow \mho$. Our picture now begins to look very similar to that pursued in 10 for the case $d=3$. There is a slight difference since in the latter case one had

$$
\begin{aligned}
& \Omega \in H^{3,0} \oplus H^{2,1} \oplus H^{1,2} \oplus H^{0,3}, \\
& \mho \in H^{0,0} \oplus H^{1,1} \oplus H^{2,2} \oplus H^{3,3} .
\end{aligned}
$$

For the K3 case however, we may only specify the general statement that both $\Omega$ and $\mho$ lie in the full cohomology $H^{*}$ at a generic point in moduli space. The following construction may be used to reconcile the approaches. Let us choose two null vectors $v$ and $w$ in $H^{*}(X, \mathbb{Z}) \cong \Lambda^{4,20}$ such that $\langle v, w\rangle=1$, so that $v$ and $w$ span a hyperbolic plane. Let $F$ be the subspace of 
$H^{*}(X, \mathbb{R})$ perpendicular to $v$ and $w$. For each oriented space-like 4-plane $\Pi$, we define

$$
\begin{aligned}
& \Omega:=\text { complex vector associated to } \Pi \cap F, \\
& \mho:=\text { complex vector associated to } \Omega^{\perp} \cap \Pi .
\end{aligned}
$$

This amounts to choosing a specific $N=(2,2)$ theory to represent an $N=(4,4)$ theory. We may now identify $F$ with $H^{2}(X, \mathbb{R})$. If $\Omega$ specifies the $H^{2,0}$ direction, (19) then implies

$$
\begin{aligned}
& \Omega \in H^{2,0} \oplus H^{1,1} \oplus H^{0,2}, \\
& \mho \in H^{0,0} \oplus H^{1,1} \oplus H^{2,2} .
\end{aligned}
$$

The complex structure of $X$ can thus be determined from $\Omega$ in the usual way. One can also obtain the Kähler form and $B$-field information from $\mho$. The mirror map interchanges the two.

Note that we may equally specify $\Omega \rightarrow \bar{\mho}, \mho \rightarrow \bar{\Omega}$ as a mirror map. We may compose this with the previous mirror map to produce the map $\Omega \rightarrow \bar{\Omega}, \mho \rightarrow \bar{\mho}$. This map may be identified as the map taking $(q, \bar{q}) \rightarrow(-q,-\bar{q})$ in the $N=(2,2)$ theory or equivalently as complex conjugation of the target space. Note that such a map is usually trivial in the moduli space of $N=(2,2)$ theories but that our construction, through specific labeling of the $U(1)$ charges, leads to a non-trivial map.

\section{Mirror Symmetry for Algebraic K3 Surfaces}

Now that we have identified how mirror maps act on the moduli space of conformal field theories on a K3 surface, we can explain several of the mirror-type phenomena which have been observed for algebraic K3 surfaces.

Given any subgroup $M$ of $H^{2}(X, \mathbb{Z})$ such that the cokernel $H^{2}(X, \mathbb{Z}) / M$ has no torsion and the intersection form on $M \otimes \mathbb{R}$ has signature $(1, \rho-1)$, we can associate the family of "algebraic K3 surfaces of type $M$ " which consists of all complex structures on the K3 for which the classes on $M$ are all of type $(1,1)$. This construction was introduced into the mathematics literature 41] as part of the explanation of Arnold's "strange duality" put forward by Pinkham and Dolgachev, and moduli spaces of these structures were studied in detail in [42, [3]. The moduli space of algebraic K3 surfaces of type $M$ has complex dimension $20-\rho$ when $M \otimes \mathbb{R}$ has signature $(1, \rho-1)$.

We can extend this idea, and define the set of "CFT's of type $M$ " on a K3 surface to be the image in the conformal field theory moduli space of the set of $\sigma$-models whose complex structure is an algebraic K3 surface of type $M$, and whose Kähler class and $B$-field are taken from the space $M \otimes \mathbb{R}$. The complexified Kähler moduli space has complex dimension $\rho$, so 
the entire CFT moduli space of type $M$ has complex dimension $(20-\rho)+\rho=20$. Since the construction segregates complex and Kähler deformations, the CFT moduli space of type $M$ embeds naturally into the $N=(2,2)$ moduli space $\mathscr{M}_{q \bar{q}}$.

Suppose that the orthogonal complement $M^{\perp}$ can be written in the form $M^{\perp}=H \oplus N$ for some lattice $N$ (orthogonal direct sum). Then a mirror map $\mu$ can be defined which exchanges the given hyperbolic plane $H$ with the hyperbolic plane $H^{0}(X) \oplus H^{4}(X)$. When $\mu$ is applied to the CFT moduli space of type $M$, it reverses the rôles of Kähler and complex structure deformations. It is not hard to see that the resulting moduli space is in fact the CFT moduli space of type $N$ - the complex structure is one for which $N$ (rather than $M$ ) consists of $(1,1)$ classes, and the Kähler structure is now taken from $N \otimes \mathbb{R}$. This gives a precise CFT interpretation of Arnold's strange duality and other related phenomena for K3 surfaces (an earlier version of which was given by Martinec [13]).

Finally we note a link between our construction of the mirror map for K3 surfaces and Voisin's construction of mirror pairs of Calabi-Yau threefolds [44] (see also [45]). The latter construction consists of taking an orbifold $Y=(X \times E) / \mathbb{Z}_{2}$, where $E$ is a torus of complex dimension one. It was shown in [44] that for suitable pairs $X_{1}, X_{2}$ of $X, Y_{1}$ and $Y_{2}$ are mirror pairs at the level of the Hodge numbers, $H^{p, q}\left(Y_{1}\right)=H^{3-p, q}\left(Y_{2}\right)$; and "A-model" and "B-model" 46] correlation functions. The K3 surfaces involved must be of type $M$, with $M$ the lattice invariant under the $\mathbb{Z}_{2}$-action. One may show that our mirror map for K3 surfaces directly establishes $X_{1}$ and $X_{2}$ as a mirror pair and thus it follows that $Y_{1}$ and $Y_{2}$ are truly mirror manifolds at the level of conformal field theory.

\section{Acknowledgements}

The work of P.S.A. was supported by NSF grant PHYS92-45317, and the work of D.R.M. was supported by an American Mathematical Society Centennial Fellowship.

\section{References}

1. P. Candelas, G. Horowitz, A. Strominger, and E.Witten, Vacuum Configuration for Superstrings, Nucl. Phys. B258 (1985) 46-74.

2. E. Witten, On the Structure of the Topological Phase of Two Dimensional Gravity, Nucl. Phys. B340 (1990) 281-332.

3. M. Bershadsky, S. Cecotti, H. Ooguri, and C. Vafa, Kodaira-Spencer Theory of Gravity and Exact Results for Quantum String Amplitudes, Harvard et al 1993 preprint HUTP-93/A025, hep-th/9309140. 
4. K. Narain, New Heterotic String Theories in Uncompactified Dimensions < 10, Phys. Lett. 169B (1986) 41-46.

5. K. Narain, M. Samadi, and E. Witten, A Note on the Toroidal Compactification of Heterotic String Theory, Nucl. Phys. B279 (1987) 369-379.

6. N. Seiberg, Observations on the Moduli Space of Superconformal Field Theories, Nucl. Phys. 303 (1988) 286-304.

7. C. Vafa, Quantum Symmetries of String Vacua, Mod. Phys. Lett. A4 (1989) 1615-1626.

8. A. Giveon and D.-J. Smit, Symmetries of the Moduli Space of (2,2) Superstring Vacua, Nucl. Phys. B349 (1991) 168-206.

9. P. S. Aspinwall and D. R. Morrison, Mirror Symmetry and the Moduli Space of K3 Surfaces, to appear.

10. P. Candelas and X. de la Ossa, Moduli Space of Calabi-Yau Manifolds, Nucl. Phys. B355 (1991) 455-481.

11. T. Eguchi, H. Ooguri, A. Taormina, and S.-K. Yang, Superconformal Algebras and String Compactification on Manifolds with SU(n) Holonomy, Nucl. Phys. B315 (1989) 193-221.

12. S. Odake, Extension of $N=2$ Superconformal Algebra and Calabi-Yau Compactification, Mod. Phys. Lett. A4 (1989) 557-568.

13. E. Martinec, Criticality, Catastrophes and Compactifications, in L. Brink et al, editor, "Physics and Mathematics of Strings", World Scientific, 1990.

14. W. Barth, C. Peters, and A. van de Ven, Compact Complex Surfaces, Springer, 1984.

15. M. de Roo, Matter Coupling to $N=4$ Supergravity, Nucl. Phys. B255 (1985) 515-531.

16. S. Cecotti, $N=2$ Landau-Ginzburg vs. Calabi-Yau Sigma Model: Nonperturbative Aspects, Int. J. Mod. Phys. A6 (1991) 1749-1814.

17. M. Berger, Sur les groupes d'holonomie homogène des variétés à connexion affine et des variétés Riemanniennes, Bull. Soc. Math. France 83 (1955) 279-330.

18. J. Wolf, Complex Homogeneous Contact Manifolds and Quaternionic Symmetric Spaces, J. Math. Mech. 14 (1965) 1033-1047.

19. G. Moore, Finite in All Directions, Yale 1993 preprint YCTP-P12-93, hep-th/9305139.

20. A. Borel and J.-P. Serre, Corners and Arithmetic Groups, Comment. Math. Helv. 48 (1973) 436-491. 
21. D. R. Morrison, Some Remarks on the Moduli of K3 Surfaces, in K. Ueno, editor, "Classification of Algebraic and Analytic Manifolds", volume 39 of Progress in Math., pages 303-332, Birkhäuser, 1983.

22. R. Kobayashi and A. N. Todorov, Polarized Period Map for Generalized K3 Surfaces and the Moduli of Einstein Metrics, Tôhoku Math. J. (2) 39 (1987) 341-363.

23. M. T. Anderson, The $L^{2}$ Structure of Moduli Spaces of Einstein Metrics on 4-Manifolds, Geom. and Funct. Analysis 2 (1992) 29-89.

24. A. Borel, Stable Real Cohomology of Arithmetic Groups, Ann. Sci. École Norm. Sup. (4) 7 (1974) 235-272.

25. P. Candelas, P. Green, and T. Hübsch, Finite Distance between Distinct Calabi-Yau Manifolds, Phys. Rev. Lett. 62 (1989) 1956-1959.

26. L. Alvarez-Gaumé and P. Ginsparg, Finiteness of Ricci-flat Supersymmetric Nonlinear $\sigma$ Models, Commun. Math. Phys. 102 (1985) 311-326.

27. C. Hull, Ultraviolet Finiteness of Supersymmetric Nonlinear Sigma Models, Nucl. Phys. B260 (1985) 182-202.

28. T. Banks and N. Seiberg, Nonperturbative Infinities, Nucl. Phys. B273 (1986) 157-164.

29. P. Candelas, T. Hübsch, and R. Schimmrigk, Relation between the Weil-Peterson and Zamolodchikov Metrics, Nucl. Phys. B329 (1990) 583-590.

30. L. Dixon, J. Harvey, C. Vafa, and E. Witten, Strings on Orbifolds, Nucl. Phys. B261 (1985) 678-686, and B274 (1986) 285-314.

31. T. Matumoto, On Diffeomorphisms of a K3 Surface, in M. Nagata et al, editor, "Algebraic and Topological Theories - to the memory of Dr. Takehiko Miyaka", pages 616-621, Kinokuniya, Tokyo, 1985.

32. C. Borcea, Diffeomorphisms of a K3 Surface, Math. Ann. 275 (1986) 1-4.

33. S. K. Donaldson, Polynomial Invariants for Smooth Four-Manifolds, Topology 29 (1990) $257-315$.

34. B. Greene and M. Plesser, Duality in Calabi-Yau Moduli Space, Nucl. Phys. B338 (1990) $15-37$.

35. D. Gepner, Exactly Solvable String Compactifications on Manifolds of $S U(N)$ Holonomy, Phys. Lett. 199B (1987) 380-388. 
36. B. Greene, C. Vafa, and N. Warner, Calabi-Yau Manifolds and Renormalization Group Flows, Nucl. Phys. B324 (1989) 371-390.

37. N. Allan, Maximality of Some Arithmetic Groups; A Note of the Arithmetic of the Orthogonal Group, An. Acad. Brasil. Ci. 38 (1966) 223-227 and 243-244.

38. R. Dijkgraaf, E. Verlinde, and H. Verlinde, On Moduli Spaces of Conformal Field Theories with $c \geq 1$, in P. DiVecchia and J. Peterson, editors, "Perspectives in String Theory", Copenhagen, 1987, World Scientific.

39. I. Piateckii-Shapiro and I. R. Shafarevich, A Torelli Theorem for Algebraic Surfaces of Type K3, Math. USSR Izvetija 5 (1971) 547-587, translation from Russian original: Izv. Akad. Nauk SSSR 35 (1971) 530-572.

40. D. Burns, Jr. and M. Rapoport, On the Torelli Problem for Kählerian K3 Surfaces, Ann. Sci. École Norm. Sup. (4) 8 (1975) 235-274.

41. H. Pinkham, Singularités exceptionnelles, la dualité étrange d'Arnold et les surfaces K-3; Groupe de monodromie des singularités unimodulaires exceptionnelles, C. R. Acad Sci. Paris Sér. A 284 (1977) 615-618 and 1515-1518.

42. D. R. Morrison, On the Moduli of Todorov Surfaces, in H. Hijikata, editor, "Algebraic Geometry and Commutative Algebra: in Honor of Masayoshi Nagata", pages 315-355, Academic Press, 1988.

43. D. R. Morrison and M.-H. Saito, Cremona Transformations and Degrees of Period Maps for K3 Surfaces with Ordinary Double Point, in T. Oda, editor, "Algebraic Geometry, Sendai 1985", volume 10 of Advanced Studies in Pure Math., pages 477-513, North-Holland, 1987.

44. C. Voisin, Miroirs et involutions sur les surfaces K3, in A. Beauville et al, editor, "Journées de Géométrie Algébrique d'Orsay", volume 218 of Astérisque, pages 273-323, Société Mathématique de France, 1993.

45. C. Borcea, K3 Surfaces with Involution and Mirror Pairs of Calabi-Yau Manifolds, Rider College 1993 preprint.

46. E. Witten, Mirror Manifolds and Topological Field Theory, in S.-T. Yau, editor, "Essays on Mirror Manifolds", International Press, 1992. 